\title{
Biomimetics: mimicking and inspired-by biology
}

\author{
Yoseph Bar-Cohen, \\ Jet Propulsion Lab, California Institute of Technology \\ 4800 Oak Grove Drive, Pasadena, CA 91109-8099, \\ yosi@jpl.nasa.gov, web: http://ndeaa.jpl.nasa.gov
}

\begin{abstract}
The evolution of nature led to the introduction of highly effective and power efficient biological mechanisms. Imitating these mechanisms offers enormous potentials for the improvement of our life and the tools we use. Humans have always made efforts to imitate nature and we are increasingly reaching levels of advancement that it becomes significantly easier to imitate, copy, and adapt biological methods, processes and systems. Advances in science and technology are leading to knowledge and capabilities that are multiplying every year. This brought us to act beyond the simple mimicking of nature. Having better tools to understand and to implement nature's principles we are now equipped like never before to be inspired by nature and to employ our tools in far superior ways. Effectively, by bio-inspiration we can have a better view and value of nature capability while studying its models to learn what can be extracted, copied or adapted. EAP as artificial muscles are adding an important element in the development of biologically inspired technologies. This paper reviews the various aspects of the field of biomimetics and the role that EAP play and the outlook for its evolution.
\end{abstract}

Keywords: Biomimetics, biologically inspired technologies, robotics, EAP, electroactive polymers

\section{INTRODUCTION}

After billions of years of evolution, nature has learned what works, what is appropriate and what would last. It also learned how to use minimum resources to achieve maximal performance and came up with numerous lasting solutions [Gordon, 1976]. Recognizing that this capability continues to be significantly ahead of many of our technologies, humans have always sought to mimic nature. This field, which is also called biomimetics, bionics, or biogenesis, has reached impressive levels that include imitating some of the human thinking process in computers by mimicking such human characteristics as making decisions and operating autonomously [Bar-Cohen, 2005]. Biology offers great model for the development of mechanical tools, computational algorithms, effective materials, as well as novel mechanisms and information technology. By adapting mechanisms and capabilities from nature, scientific approaches have helped humans understand the related phenomena and the associated principles in order to engineer novel devices and improve their capability. The cell-based structure, which makes up the majority of biological creatures, offers the ability to grow with fault-tolerance and self repair, while doing all of the things that are characterizing biological systems. Therefore, engineering structures that are made of multiple cells would allow for the design of devices and mechanisms that are impossible with today's capabilities. Emerging nano-technologies are increasingly enabling the potential of such capabilities. Some of the commercial implementations of the progress in biomimetics can be seen in toy stores, in which toys appear and behave like living creatures (e.g., dogs, cats, birds, and frogs). More serious benefits of biomimetics include the development of prosthetic implants that appear very much like biological origin, and sensory aiding mechanisms that are interfaced to the brain to assist in hearing or seeing or controlling instruments.

The subject of copying, imitating, and learning from biology was coined Bionics by Jack Steele, of the US Air Force in 1960 at a meeting at Wright-Patterson Air Force Base in Dayton, Ohio [Vincent, 2001] and Otto H. Schmitt coined the term Biomimetics in 1969 [Schmitt, 1969]. This field is increasingly identified with emerging subjects of science and engineering. The term itself is derived from bios, meaning life, and mimesis, meaning to imitate. This new science represents the studies and imitation of nature's methods, designs and processes. While some of nature's basic configurations and designs can be copied, there are many ideas from nature that are best adapted if they are to serve as inspiration using human-made capabilities.

Through evolution, nature has "experimented" with various solutions to its challenges and has improved the successful solutions. Organisms that nature created, which are capable of surviving, are not necessarily optimal for their technical performance. Effectively, all they need to do is to survive long enough to reproduce. Living systems archive 
the evolved and accumulated information by coding it into the species' genes and passing the information from generation to generation through self-replication. Thus, through evolution nature, or biology, has experimented with the principles of physics, chemistry, mechanical engineering, materials science, mobility, control, sensors, and many other fields that we recognize as science and engineering. The process has also involved scaling from nano and macro, as in the case of bacteria and virus, to the macro and mega, including our life scale and the dinosaurs, respectively. While there is still doubt regarding the reason why creatures such as the mammoth went extinct, one may argue that the experiment in the evolution of mega-scale terrestrial biology failed. While marine creatures such as the whales survived, the experiment with large size terrestrial biology ended with the extinction of the prehistoric mega creatures (e.g., dinosaurs and mammoths). Such creatures can now be found only in excavation sites and natural history museums.

\section{BIOLOGY AS A MODEL}

Nature has an enormous pool of inventions that passed the harsh test of practicality and durability in changing environment. In order to harness the most from nature's inventions it is critical to bridge between the fields of biology and engineering. This bridging effort can be a key to turning nature's inventions into engineering capabilities, tools, and mechanisms. In order to approach nature in engineering terms it is necessary to sort biological capabilities along technological categories using a top down structure or vise versa. Namely, one can take each aspect of the biologically identified characteristics and seek an analogy in terms of an artificial technology. The emergence of nano-technologies, miniature highly capable and fast micro-processors, effective power storage, large compact and fast access memory, wireless communication and many others are making the mimicking of nature capabilities significantly more feasible. One reason for this is because both natural and artificial structures depend on the same fundamental units of atoms and molecules.

There are many characteristics that can identify a biomimetic mechanism and an important one is the capability to operate autonomously in complex environments, being adaptable to unplanned and unpredictable changes and perform multifunctional task. Making mechanism with such characteristics dramatically increases the possible capabilities and can reach levels that can be as good or superior to human/animals. This may include operating for 24-hours a day without a break or operating in conditions that pose health risks to humans. Benefits from such capabilities can include performing security monitoring and surveillance, search and rescue, operating under chemicals, biological and nuclear hazards, taking immediate corrective and warning actions as well as many others that are only limited by our imagination. Some of the biologically inspired capabilities that are/can be implemented into effective mechanisms include:

- Multifunctional materials and structures

- High strength materials and configurations

- Just-in-time manufacturing

- Deployable structures adapting the unfolding leaves from the bud.

- Hammering without vibration back-propagation as the woodpecker does

- Nano-structures mimicking full systems like the virus and bacteria or making cell based structures

- Robots that operate cooperatively and have biomimetic behavior as well as body expression for communication

- Mimicking aerodynamic performance of the dragon fly and the hummingbird

- Biomimetic based sensors and feedback

- Optimization tools and algorithms

- Multifunctional Mobility that combines walking, crawling, climbing (trees, cliffs, or walls), jumping and leaping, swimming, flying, grasping, digging, and manipulating objects.

- Attaching to steep walls and upside down from a ceiling (see Figure 1)

- Autonomous locomotion

\subsection{Artificial Life}

The name Artificial Life (A-Life) suggests the synthesizing of life from non-living components [Adami, 1998]. A-Life is dedicated to the investigation of scientific, engineering, philosophical, and social issues involved in our rapidly increasing technological capability to synthesize from scratch life-like behaviors using computers, machines, molecules, and other alternative media [Langton, 1995]. Artificial Life focuses on the broad characteristics of biology and contributes to the development of machines that evolve, sociable robots, artificial immune systems that protect computers from malicious viruses, and virtual creatures that learn, breed, age, and die. Moreover, biologists can now 
study evolution in virtual worlds, and medical students and doctors can study the operation mechanisms of various living organs, including the heart with its cells, enabling to learn in ways that are impossible to do with actual living organs.

FIGURE 1: A 4-legged robot called STAR (Steep Terrain Access Rover) is under development at JPL [Courtesy of Brett Kennedy, JPL]

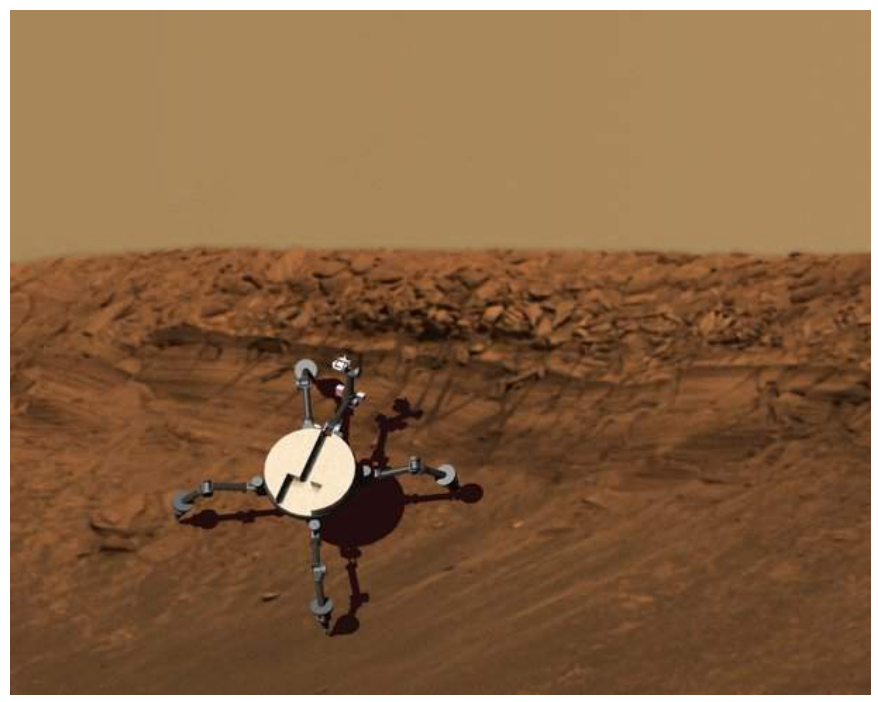

\subsection{Artificial Intelligence (AI)}

According to the American Association for Artificial Intelligence (AAAI), artificial intelligence (AI) is, "the scientific understanding of the mechanisms underlying thought and intelligent behavior and their embodiment in machines". AI is a branch of computer science that studies the computational requirements for such tasks as perception, reasoning, and learning, to allow the development of systems that perform these capabilities [Russell and Norvig, 2003]. AI researchers are addressing a wide range of problems that include studying the requirements for expert performance of specialized tasks, explaining behaviors in terms of low-level processes, using models inspired by the computation of the brain and explaining them in terms of higher-level psychological constructs such as plans and goals. The field seeks to advance the understanding of human cognition, understand the requirements for intelligence in general, and develop artifacts such as intelligent devices, autonomous agents, and systems that cooperate with humans to enhance their abilities. The name AI was coined in 1956, though the roots of the field may be attributed to the efforts in World War II to crack enemy codes by capturing human intelligence in a machine that was called Enigma. This approach eventually led to the 1997 computer success of IBM's Deep Blue in beating the world-champion chess player Garry Kasparov. Even though this was an enormous success for computers, it is still unlikely to produce a tool that resembles human intelligence. AI technologies consist of an increasing number of tools, including artificial neural networks, expert systems, fuzzy logic, and genetic algorithms [Luger, 2001].

\subsection{Structures and tools}

Biological creatures can build amazing shapes and structures using materials in their surroundings or materials that they produce. The produced shapes and structures within a species are very close copies. They are also quite robust and support the structure's required function over the duration that it is needed. Such structures include the birds' nest and the bees' honeycomb. Often the size of a structure can be significantly larger than the species that built it, as is the case with the spider's web (Figure 2). One creature that has a highly impressive engineering skill is the beaver, which constructs dams as its habitat on streams. Other interesting structures include underground tunnels that gophers and rats build. Birds make their nest from twigs and other materials that are secured to various stable objects, such as trees, and their nests are durable throughout the bird's nesting season. Many nests are hemispherical in the area where the eggs are laid. One may wonder how birds have the capability to design and produce the correct shape and size that matches the requirements of allowing laid eggs to hatch and grow as chicks until they leave the nest. The nest's size accounts for the potential number of eggs and chicks, in terms of required space. Even plants offer engineering inspiration, where mimicking the concept of seeds that adhere to an animal's fur, Velcro was invented and has led to an enormous impact in many fields, including clothing and electric-wires strapping. Because of their intuitive characteristics, the use of biologically-based rules allows for the making of devices and instruments that are user friendly where humans can figure out how to operate using minimal instructions. 


\subsection{Materials and processes in biology}

The body is a chemical laboratory that processes chemicals acquired from nature and turns them to energy, construction materials, waste and various multifunctional structures [Mann, 1995]. Natural materials have been well recognized by humans as sources of food, clothing, comfort, and many others where, to name few, one can include fur, leather, honey, wax, milk and silk. Even though some of the creatures and insects that produce materials are relatively small, they can produce quantities of materials that are sufficient to meet human consumption on a scale of mass production (e.g., honey, silk and wool). The use of natural materials can be traced back thousands of years. Silk, which is produced to protect the cocoon of the silkmoth, has great properties that include beauty, strength and durability. These advantages are well recognized by humans and the need to make them in any desired quantity led to the production of artificial versions and imitations. Some of the fascinating capabilities of natural materials include self-healing, self replication, reconfigurability, chemical balance, and multifunctionality. Many man-made materials are processed by heating and pressurizing, and this is in contrast to nature which always uses ambient conditions. Materials, such as bone, collagen or silk are made inside the organism's body without the harsh treatment that is used to make our materials. The fabrication of biologically derived materials produces minimum waste and no pollution, where the result is biodegradable and is recycled by nature. Learning how to process such materials can make our material choices greater and improve our ability to create recyclable materials that can better protect the environment. There are also studies that are improving prosthetics, which include hips, teeth, structural support of bones and others.

FIGURE 2: The spider constructs an amazing web that is made of silk material that for a given weight it is 5 times stronger that steel (The photograph was taken in a foggy day making it easier to see the web due to the droplets)

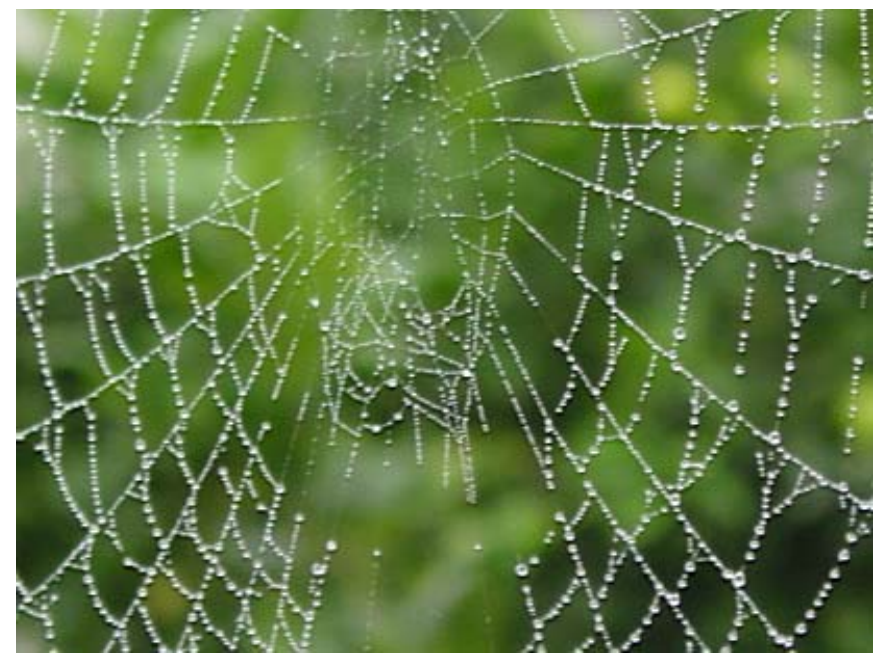

\subsection{Artificial Muscles}

Polymers that can be stimulated to change shape and size have been known for years. The functional similarity of such polymers led to their being named artificial muscles. The activation mechanism for such polymers include electric, chemical, pneumatic, optical, and magnetic. Electrical excitation is one of the most attractive stimulators that can produce elastic deformation in polymers. The convenience and the practicality of electrical stimulation, as well as the improved capabilities, make the electroactive polymers (EAP) one of the most attractive among the activatable polymers [Bar-Cohen 2001 and 2004].

Generally, EAP materials can be divided into two major categories based on their activation mechanism: electronic and ionic. Most electronic polymers (electrostrictive, electrostatic, piezoelectric, and ferroelectric) require high activation fields $(>150 \mathrm{~V} / \mu \mathrm{m})$ close to the breakdown level. However, they can be made to hold the induced displacement under activation of a DC voltage, allowing them to be considered for robotic applications. These materials have a faster response, a greater mechanical energy density, and they can be operated in air. In contrast, ionic EAP materials (gels, IPMC, conductive polymers, and carbon nanotubes) require drive voltages as low as $1-5 \mathrm{~V}$ and produce significant bending. However, bending actuators have relatively limited applications for mechanically demanding tasks due to the low force or torque that can be induced. Also, with some exceptions, these materials require maintaining their wetness and when containing water they suffer electrolysis with irreversible effects when they are subjected to voltages above 1.23-V. Except for conductive polymers, it is difficult to sustain DC-induced displacements. 
Unfortunately, EAP-based actuators, are still exhibiting low force below their efficiency limits, are not robust, and are not available as commercial materials for practical application considerations. Each of the known materials requires adequate attention to the associated unique properties and constraints. In order to be able to take these materials from the development phase to use as effective actuators, there is a need to have an established EAP infrastructure. Effectively addressing the requirements of the EAP infrastructure involves developing its science and engineering basis; namely having an adequate understanding of EAP materials' behavior, as well as processing and characterization techniques. Enhancement of the actuation force requires understanding the basic principles, computational chemistry models, comprehensive material science, electromechanical analysis and improved material processing techniques. Efforts are made to gain a better understanding of the parameters that control the EAP electro-activation force and deformation. The processes of synthesizing, fabricating, electroding, shaping, and handling are being established and refined to maximize the EAP materials actuation capability and robustness. In addition, methods of reliably characterizing the response of these materials are being developed. This effort also includes the establishment of a database with documented material properties in order to support design engineers who are considering the use of these materials. Various configurations of EAP actuators and sensors are being modeled to produce an arsenal of effective, smart EAP-driven systems. The development of the infrastructure is multidisciplinary and is requiring international collaboration and these efforts are currently well underway worldwide.

In 1999, the author challenged the world's research and engineering community to develop a robotic arm that is actuated by artificial muscles (moniker for electroactive polymers (EAP)) to win a wrestling match against a human opponent. The match's objectives are to promote advances towards making EAP actuators that are superior to the performance of human muscles. Also, it is sought to increase the worldwide visibility and recognition of EAP materials; attract interest among potential sponsors and users; and lead to general public awareness since it is hoped that they will be the end users and beneficiaries in many areas including medical, commercial, and military.

\subsection{Bio-Sensors}

Living creatures are equipped with a sensory system, which provides input to the central nervous system about the environment around and within their body and the muscles are commanded to action after analysis of the received information [Hughes, 1999]. Biological sensory systems are extremely sensitive and limited only by quantum effects [Bialek, 1987]. This sensory network is increasingly imitated, where we find our surrounding filled with sensors. Such sensors are monitoring our property from intruders; releasing soap and water when washing our hands; releasing hot air or paper towels to dry our hands; tracking our driving speed; observing our driving through intersections that are monitored by traffic lights; as well as many other applications. Our cars sense when we close the doors, whether there is sufficient air in the tires, charge in the battery and oil in the engine, and if all the key functions are operating properly. Sensors also control the flow of gasoline to the ignition system in our cars to optimize gas consumption. Similar to the ability of our body to monitor the temperature and keep it within healthy acceptable limits, our habitats, working, and shopping areas have environment control to provide us with comfortable temperatures. These examples are only a small number of the types of sensors that are used in our today's surroundings and the instruments that we use. Pressure, temperature, optical and acoustical sensors are widely in use and efforts are continuously being made to improve their sensing capability and reduce their size and the required power while mimicking ideas from biology. These include adapting principles from the eyes to camera, the whiskers of rodents as sensors for collision avoidance, and acoustic detectors that imitate the sonar in bats.

\section{PRESENT TECHNOLOGY, FUTURE POSSIBILITIES AND POTENTIALS}

Developing biomimetic mechanisms requires employing many disciplines, tools and capabilities. It involves materials, actuators, sensors, structures, control, and autonomous operations. Mimicking nature has immensely expanded the collection of tools that are available to us in performing tasks that once were considered science fiction. As technology evolves, increasing numbers of biologically inspired mechanisms and functions that emulate the capability of creatures and organisms are expected to emerge. The challenges to making such biomimetic technologies that are copied or adapted depend on the complexity that is involved. Examples can include making marine vehicles that mimic the shark skin of having low friction surface in water or the use of anti-freeze proteins that are found in some marine creatures allowing them to sustain temperatures below freezing.

One of the emerging areas of biomimetics is the artificial muscles, which are the moniker for electroactive polymers (EAP). It offers enormous potential for many aspects of our life. The easy capability to produce EAP materials in various shapes can be exploited to making future mechanisms and devices using such methods as stereolithography and 
ink-jet printing techniques. A polymer can be dissolved in a volatile solvent and ejected drop-by-drop onto various substrates. Such processing methods offer the potential of making systems and robots in full 3D details that include the EAP actuators allowing rapid prototyping and quick mass production [Bar-Cohen, 2004]. A possible vision for such technology can be the fabrication of insect like robots that can be made to fly and pack themselves into a box to be ready for shipping once they are made. Other example can be the rapid prototyping of robots with controlled characteristics that follow specific movie scripts with the appearance and behavior of the desired artificial actors. The robots appearance and behavior can be modified rapidly as needed for the evolving script and when changes need to be made the artificial actors can be rapidly produced in any desired modification. Further, making insect-like robots may help inspecting hard to reach areas of aircraft structures where these miniature robots can be launched to conduct the required inspection procedures and then download the information about the structure integrity upon exiting the concealed area. Using effective EAP actuators to mimic nature would immensely expand the collection and functionality of devices and mechanisms that are currently available. Important addition to this capability can be the application of tele-presence combined with virtual reality using haptic interfaces. While such capabilities are expected to significantly change future robots, additional effort is needed to develop robust and effective EAP-based actuators.

Considering the current limitations of artificial muscles and their capability to support biomimetic applications the author posed a challenge to the worldwide science and engineering community to develop a robotic arm that is actuated by artificial muscles to win an arm wrestling match against a human opponent (Figure 3) [Bar-Cohen, 2004]. The first competition is planned to be held on March 7, 2005 during the EAP Session of the SPIE's EAP Actuators and Devices (EAPAD) Conference, which is part of the Smart Structures and Materials Symposium. Progress towards making such robotic arms win a match against human will lead to significant benefits, particularly in the medical area, including effective prosthetics. A remarkable contribution would be to one day seeing a disabled person jogging to the grocery store using this technology. It would lead to exciting new generations of robots that can change our daily life with the possibility of robots that are a household assistant and intelligent companion substituting the dog as "man's best friend". Another important benefit that may be achieved with the success in winning this challenge would be a milestone demonstration of the capability to produce superior biomimetic robots.

FIGURE 3: Grand challenge for the development of EAP actuated robotics.

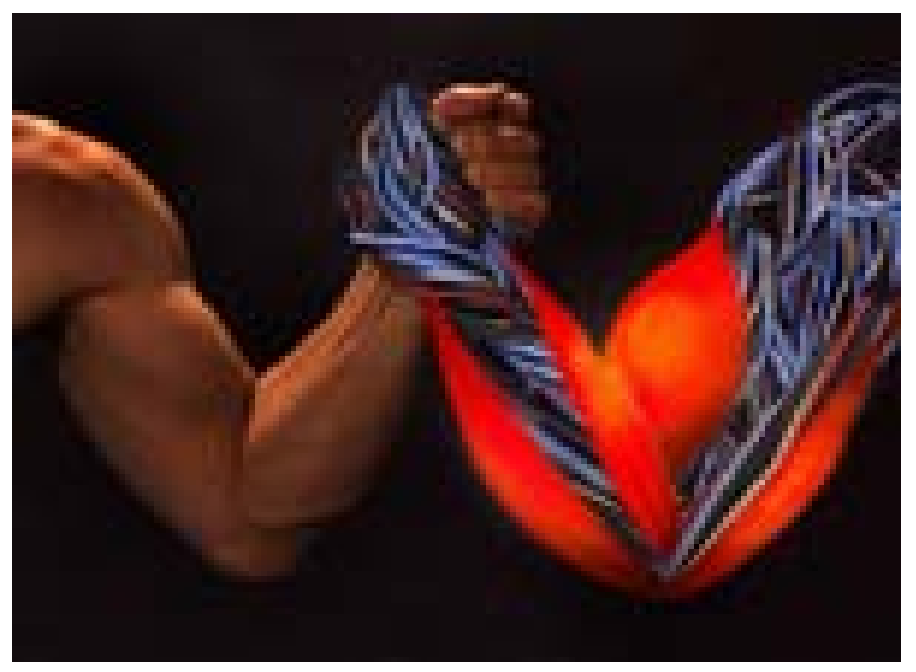

Availability of strong and robust artificial muscles may enable in future years to produce such biomimetic legged robots that can run as fast as a cheetah, carry mass like a horse, climb steep cliffs like a gecko, reconfigure its body like an octopus, fly like a bird and dig tunnels like a gopher. This can be an incredible vision for robots that can potentially be used in future exploration of planets in the universe. This can lead to future NASA mission plans that may include a script for the robots operation that may follow science fiction ideas. Hopefully, these robots would be able to operate autonomously to detect water, various resources, and possibly biological indicators in the search for past or present life or even construct facilities for future human habitats.

\section{AREAS OF CONCERNS AND CHALLENGES TO BIOMIMETICS}

There many examples of concepts, devices, mechanisms and others which were mimicked or inspired by biology. One of the amazing capabilities of nature include the spider ability to create just in time, in room temperature and pressure, 
an incredibly flat and strong web structure that is quite durable in outdoor conditions. The spider web may have inspired the fishing net, the fibers, the fabric of the clothing that we wear, and many other things that we use in our daily life. In some cases, the possibilities seen in nature allowed us to make far superior capabilities. For example, with flying, the human efforts to copy birds' wings in order to produce a flying machine let to quite limited capability as was demonstrated in the late 1880's and 1890's by Horatio Phillips and Otto Lilienthal. Only after we mastered aerodynamic principles did we manage to fly with great superiority over birds. The aircraft capabilities that engineers are making are an incredible human success in which our engineered aircraft far exceed any flying creature that ever existed. This includes reaching significantly higher and faster, carrying way more weight using aircraft that have enormous volume as we can see in airports today. The only thing airplanes can't do yet is perch on a wire (though microplanes now under development may end up doing just that).

Some human inventions that appear biomimetic may have not necessarily been the result of an actual adaptation of nature ideas [Altshuller, 1988]. The process of innovation and introduction of invention by humans as problem solvers can be difficult to trace. In some tools, nature may not have been the immediate model, and similarities may just be a coincident. However, some human inventions have been admittedly mimicked from biology, as in the case of Velcro. In the case of honeycomb, even if it may not have been the direct source of this invention that is used in many aircraft structures [Gordon, 1976], it is still the same structure and its name is the same as the product that is made by the bees. The potential of reinventing nature's innovation may be reduced if these inventions can be documented, not as biological observations but as engineering mechanisms and tools. Effectively, there is a need to establish a database and handbooks that logically catalogues nature's capabilities, specifications, mechanisms, processes, tools and functions in terms of principles, materials, dimensions, limitations, etc. Such documented information, which can be produced by biologists for use by engineers, may greatly help humans in making novel biomimetic inventions. Working towards such an objective, one can consider nature in technology terms, while possibly considering the use of a unified approach to describe biological inventions. Such documentation might help to accelerate advances in human made technologies.

The December 2004 tsunami disaster caused over two hundred thousand casualties and led to million homeless people, where in contrast very few animals died. This fact suggests that humans have lost the ability to sense such nature dangers. It is difficult to believe that such a sensing capability can be reacquired by humans and therefore alternative detection techniques are needed. Most countries do not have the required monitoring system due to the very high associated cost and the fact that such disaster may occur once in tens or hundreds of years. Adapting the sensing capability of animals would potentially lead to affordable detection technology.

Nature offers many capabilities that are unique to some species, and understanding the requirements for their adaptation can help us in many ways. Some of these capabilities are still mysteries that offer enormous potential for humans. For medical applications, one may wonder about bears' capability to sleep for 6 months without urinating and without poisoning its blood. Learning the clues to this capability may help fight diabetes. The ability of the lizard to drop its tail as a decoy in case of danger, and growing it back without scars is an important model for the field of medicine. Adapting this capability can help heal the disabled and severely injured.

\section{CONCLUSIONS}

After billions of years of evolution, nature developed inventions that work, which are appropriate for the intended tasks and that last. The evolution of nature led to the introduction of highly effective and power efficient biological mechanisms. Failed solutions often led to the extinction of the specific species that became a fossil. In its evolution, nature archived its solutions in genes of creatures that make up the terrestrial life around us. Imitating nature's mechanisms offers enormous potentials for the improvement of our life and the tools we use. Humans have always made efforts to imitate nature and we are increasingly reaching levels of advancement where it becomes significantly easier to mimic biological methods, processes and systems. Advances in science and technology are leading to knowledge and capabilities that are multiplying every year. These improvements led to capabilities to better understand and implement nature's principles in more complex ways. Effectively, we have now significantly better appreciation of nature's capabilities allowing us to employ, extract, copy and adapt its inventions.

Benefits from the study of biomimetics can be seen in many applications, including stronger fiber, multifunctional materials, improved drugs, superior robots, and many others. Another aspect of biomimetics is to recognize the importance of protecting species from extinction, lest we lose Nature's solutions that managed to survive, but that we have not yet studied or still don't understand. Nature offers a model for us as humans in our efforts to address our needs. We can learn manufacturing techniques from animals and plants such as the use of sunlight and simple compounds to produce with no prolusion, biodegradable fibers, ceramics, plastics, and various chemicals. Nature has already provided 
a model for many human-made devices, processes and mechanisms. One can envision the emergence of extremely strong fibers that are woven as the spider does, and ceramics that are shatterproof emulating the pearl. Besides providing models, nature can serve as a guide to determine the appropriateness of our innovations in terms of durability, performance, and compatibility. Biomimetics has many challenges, where the author's armwrestling challenge announced in 1999 has taken the human muscle as a baseline for the development of artificial muscles.

The inspiration of nature is expected to continue leading to technology improvements and the impact is expected to be felt in every aspect of our lives. Some of the solutions may be considered science-fiction in today's capability, but as we improve our understanding of nature and develop better capabilities this may become a reality that is closer than we think.

\section{ACKNOWLEDGEMENT}

Research reported in this manuscript was conducted at the Jet Propulsion Laboratory (JPL), California Institute of Technology, under a contract with National Aeronautics and Space Administration (NASA).

\section{REFERENCE}

Adami C., Introduction to Artificial Life, ISBN 0-387-94646-2, Springer-Verlag, Berlin, Germany (1998), pp. 1-374. Altshuller G., Creativity as an Exact Science, Gordon and Breach, NY (1988).

Bar-Cohen Y. (Ed.), "Electroactive Polymer (EAP) Actuators as Artificial Muscles - Reality, Potential and Challenges," ISBN 0-8194-4054-X, SPIE Press, Bellingham, WA, USA, Vol. PM98, (March 2001), pp. 1-671.

Bar-Cohen Y., (Ed.), "Biomimetics: Mimicking and being Inspired by Biology,” CRC Press, to be published in 2005.

Bar-Cohen Y., "Electroactive Polymer (EAP) Actuators as Artificial Muscles - Reality, Potential and Challenges," 2nd Edition, ISBN 0-8194-5297-1, SPIE Press, Bellingham, WA, USA, Vol. PM136, (March 2004), pp. 1-765

Bialek W, "Physical limits to sensation and perception," Annual Review of Biophysics, Biophysics Chemistry. Vol. 16, (1987), pp. 455-478

Gordon, J.E. "The New Science of Strong Materials, or Why You Don't Fall Through the Floor” 2nd Ed. London: Pelican-Penguin, ISBN: 0140209204, (1976) pp. 1-287.

Hughes H. C., "Sensory Exotica A World Beyond Human Experience, ISBN 0-262-08279-9, MIT Press, Cambridge, MA, 1999. pp. 1-359

Langton C. G. (Ed.), “Artificial Life: An Overview,” ISBN: 0262621126, MIT Press (1995), pp. 1- 336.

Luger G. F., Artificial Intelligence: Structures and Strategies for Complex Problem Solving ISBN: 0201648660, Pearson Education Publishers (2001), pp. 1-856.

Mann S. (Ed.), Biomimetic Materials Chemistry, Wiley Publishers, ISBN: 0-471-18597-3, (1995) pp. 1-400.

Russell S. J., P. Norvig, Artificial Intelligence: A Modern Approach, Pearson Education, ISBN: 0137903952, (2003), pp. 1- 1132.

Schmitt O. H., "Some Interesting and Useful Biomimetic Transforms," Proceeding, Third International Biophysics Congress, Boston, Mass., Aug. 29-Sept. 3,1969, p.297.

Vincent J. F. V., "Stealing Ideas from Nature," Chapter 3 in Deployable structures, Springer-Verlag, Vienna, Pellegrino S. (Ed.), 2001, 51-58 